ANh/mso/CP-101980

\title{
Investigations of Chemical Vapor Deposition of GaN Using Synchrotron
} Radiation

C. Thompson ${ }^{\mathrm{a}, \mathrm{b}}$, G. B. Stephenson ${ }^{\mathrm{b}}$, J.A. Eastman ${ }^{\mathrm{b}}$, A. Munkholm ${ }^{\mathrm{c}}$, O. Auciello ${ }^{\mathrm{b}}$, M.V. Ramana Murty $^{b}$, P. Fini, ${ }^{d}$, S.P. DenBaars ${ }^{d}$, and J.S. Speck ${ }^{d}$

${ }^{2}$ Department of Physics, Northern Illinois University, DeKalb, IL 60115 USA

${ }^{b}$ Materials Science Division, Argonne National Laboratory, Argonne, IL 60439 USA

${ }^{\circ}$ Chemistry Division, Argonne National Laboratory, Argonne, IL 60439 USA

${ }^{\mathrm{d}}$ Materials Department, University of CA, Santa Barbara, CA 93106 USA

\section{Materials Science Division \\ Argonne National Laboratory}

Argonne, IL 60439

The submitted manuscript has been created by
the University of Chicago as Operator of Argonne
National Laboratory ("Argonne") under Contract
No. W-31-109-ENG-38 with the U.S. Department
of Energy. The U.S. Government retains for
itself, and others acting on its behalf, a paid-up,
non exclusive, irrevocable worldwide license in
said article to reproduce, prepare derivative
works, distribute copies to the public, and
perform publicly and display publicly, by or on
behalf of the Govemment.

May 2000

/jc

Distribution:

1-2. PRS, 203

3. J. M. Gibson

4. P. A. Montano

5. F. Y. Fradin

6. Office of Science

7. J. Coble

8. Authors

Submitted as an invited talk and to be published in the Conference Proceedings of the $197^{\text {th }}$ Meeting of The Electrochemical Society, Toronto, Canada, May 14-18, 2000.

This work is supported by the National Science Foundation under grant DMR-9704201, the State of IL under HECA and the U.S. Department of Energy, BES-DMS under contract No.W-31-109-ENG-38. 


\section{DISCLAIMER}

This report was prepared as an account of work sponsored by an agency of the United States Government. Neither the United States Government nor any agency thereof, nor any of their employees, make any warranty, express or implied, or assumes any legal liability or responsibility for the accuracy, completeness, or usefulness of any information, apparatus, product, or process disclosed, or represents that its use would not infringe privately owned rights. Reference herein to any specific commercial product, process, or service by trade name, trademark, manufacturer, or otherwise does not necessarily constitute or imply its endorsement, recommendation, or favoring by the United States Government or any agency thereof. The views and opinions of authors expressed herein do not necessarily state or reflect those of the United States Government or any agency thereof. 


\section{DISCLAIMER}

Portions of this document may be illegible in electronic image products. Images are produced from the best available original document. 


\title{
INVESTIGATIONS OF CHEMICAL VAPOR DEPOSITION OF GaN USING SYNCHROTRON RADIATION
}

\author{
Carol Thompson ${ }^{a, b}$, G.B. Stephenson ${ }^{b}$, J.A. Eastman ${ }^{b}$, A. Munkholm ${ }^{c}$, O. Auciello ${ }^{b}$, \\ M.V. Ramana Murty ${ }^{b}$, P. Fini ${ }^{d}$, S. P. DenBaars ${ }^{d}$, and J. S. Speck ${ }^{d}$ \\ ${ }^{a}$ Department of Physics, Northern Illinois University, DeKalb, IL 60115 USA \\ ${ }^{b}$ Materials Science Division, Argonne National Laboratory, Argonne, IL 60439 USA \\ ${ }^{c}$ Chemistry Division, Argonne National Laboratory, Argonne, IL 60439 USA \\ ${ }^{d}$ Materials Department, University of California, Santa Barbara, CA 93106 USA
}

\section{Abstract}

We apply synchrotron $\mathrm{x}$-ray analysis techniques to probe the surface structure of $\mathrm{GaN}$ films during synthesis by metal-organic chemical vapor deposition (MOCVD). Our approach is to observe the evolution of surface structure and morphology in real time using grazing incidence $\mathrm{x}$-ray scattering (GIXS). This technique combines the ability of $\mathrm{x}$-rays to penetrate the chemical vapor deposition environment for in situ measurements, with the sensitivity of GIXS to atomic scale structure. In this paper we present examples from some of our studies of growth modes and surface evolution as a function of process conditions that illustrate the capabilities of synchrotron x-ray analysis during MOCVD growth. We focus on studies of the homoepitaxial growth mode, island coarsening dynamics, and effects of impurities.

\section{A. INTRODUCTION}

Although most in situ studies of atomic-scale surface structure and morphology during epitaxial growth have been performed in vacuum environments, chemical interactions between the vapor-phase environment and the growing surface may play a critical role during chemical vapor deposition (CVD). For example, the competition between deposition, surface diffusion, and nucleation and propagation of atomic steps on the surface determines the surface morphology during crystal growth, in particular the homoepitaxial growth mode [1]. It is clear that these rates will be affected by the surface chemistry and structure as determined by process parameters such as gas composition, flow rate, pressure, substrate temperature, and impurity level. Real-time observation of surface morphology as a function of process conditions could greatly aid in understanding these complex interactions. However, there have been few in situ measurements of atomic-scale surface structure during CVD growth because few surface analysis techniques are compatible with this reactive vapor phase 
environment. Our approach has been to observe the evolution of surface structure and morphology in real time using grazing incidence x-ray scattering (GIXS). This technique combines the ability of $\mathrm{x}$-rays to penetrate the CVD environment for in situ measurements, with the sensitivity of GIXS to atomic scale structure. We have applied this technique to investigating the epitaxial growth of GaN by metal-organic chemical vapor deposition (MOCVD). Our studies have included in situ measurements of surface structure in the MOCVD environment [2,3], real-time measurements of growth modes [4], faceting transitions [5], effects of impurities on growth [6], and wing tilt during lateral epitaxial overgrowth [7].

In this paper we present examples from some of our studies of growth modes and surface evolution as a function of process conditions, which illustrate the capabilities of synchrotron x-ray analysis during MOCVD growth. In particular, we report on studies of the homoepitaxial growth mode, island coarsening dynamics, and effects of impurities.

\section{B. EXPERIMENTAL}

Real-time $x$-ray measurements are carried out using a vertical-flow MOCVD chamber mounted on a "z-axis" surface diffractometer designed for in situ GIXS studies [8], located at the BESSRC undulator beamline 12-ID-D at the Advanced Photon Source (Fig. 1). A relatively high x-ray energy of $24 \mathrm{keV}(0.517 \AA)$ is used to penetrate the 2 -mm-thick quartz walls of the chamber. In addition to the x-ray probe, we also use an in situ normal-incidence optical reflectance probe for determining film thickness and macroscopic roughness by interferometry. Homoepitaxial growth is performed on the $\mathrm{GaN}(0001)$ (Ga-face) surface using trimethylgallium (TMG) and ammonia $\left(\mathrm{NH}_{3}\right)$ precursors, at substrate temperatures up to $1050^{\circ} \mathrm{C}$. We also explore the effect of silicon and oxygen impurities on GaN film growth by introducing disilane and $\mathrm{O}_{2}$. The carrier gas is nitrogen, which is also used as the window and heater purge. The sample temperature is obtained to within $\pm 5^{\circ} \mathrm{C}$ by calibrating it with respect to the heater thermocouple using optical reflectometry to observe the thermal expansion of a sapphire substrate.

To observe atomic-scale changes in surface morphology during growth, we measure features in the scattering pattern known as crystal truncation rods (CTRs) [9]. These are streaks of $x$-ray scattering intensity extending through the Bragg peaks of the bulk crystal in a direction normal to the crystal surface. CTR intensities measured with $\mathrm{x}$-rays can be directly related to the morphology of the crystal surface since the scattering is kinematic. In particular, at "anti-Bragg" positions, where each atomic plane of the crystal scatters out-of-phase with its neighbors, the x-ray CTR intensity is a sensitive measure of atomic-scale roughness. The evolution of the CTR intensity with time during growth can be used to identify the homoepitaxial growth mode of the crystal. For step-flow mode, in which atoms arriving on the surface attach at existing step edges, the surface morphology is in steady state and the CTR intensity stays constant. For layer-by-layer mode, in which islands are nucleated on 


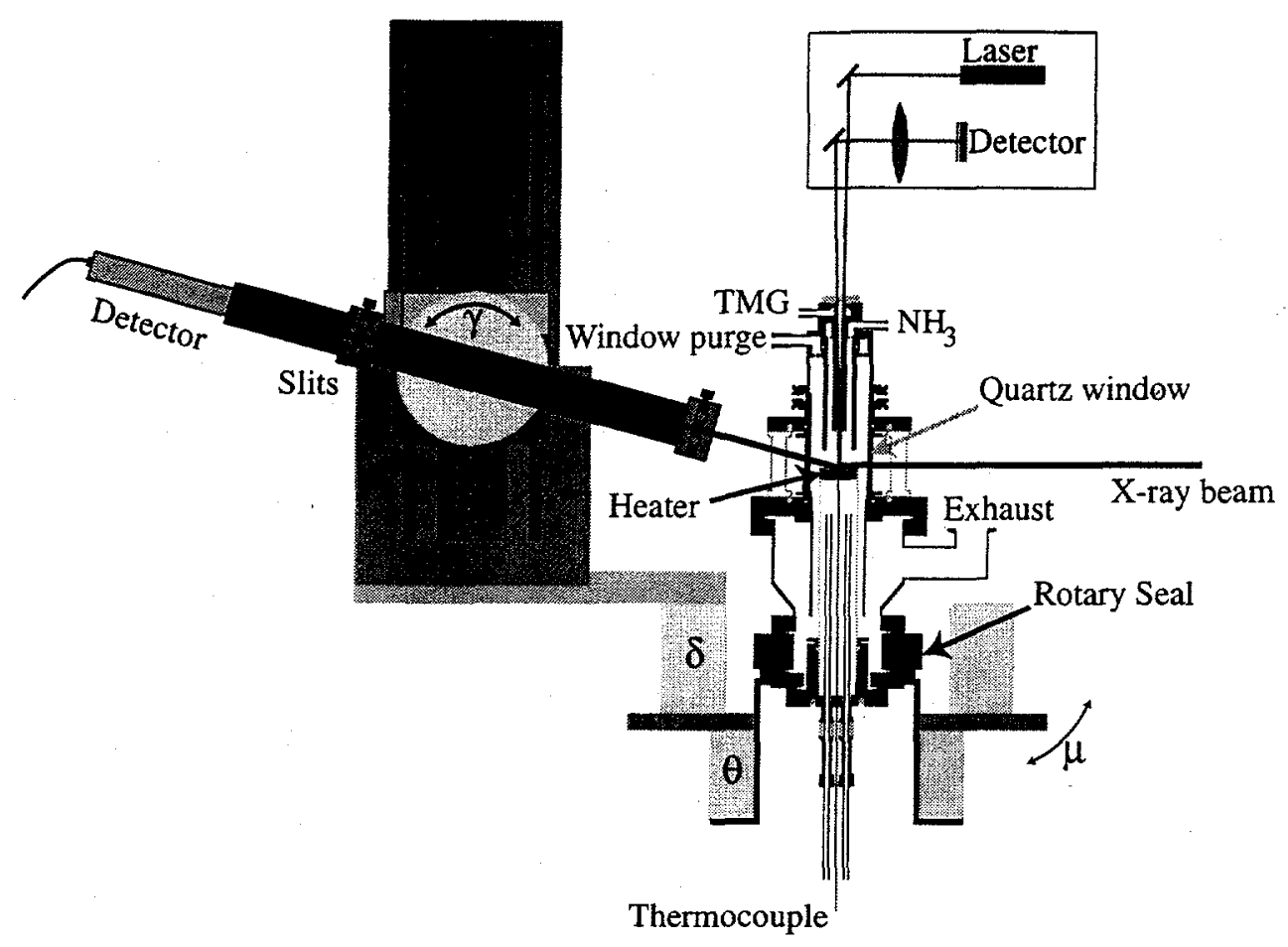

FIG. 1. Schematic of vertical flow MOVPE chamber installed on beamline 12-ID-D at the Advanced Photon Source.

the terraces between steps and subsequently coalesce, the CTR intensity oscillates with each monolayer of growth $(1 \mathrm{ML}=2.6 \AA$ for $\mathrm{GaN})$ due to the transient "roughness" (cyclic changes in the occupations of neighboring atomic planes) induced by the island formation. For 3-D mode, in which deposited atoms typically remain on the layer where they arrive, the surface roughens rapidly and causes the CTR intensity to decay monotonically.

\section{HOMOEPITAXIAL GROWTH MODES}

For studies of homoepitaxy, we deposit onto "templates" consisting of $\sim 2-\mu \mathrm{m}$ thick films of device-quality (0001) hexagonal GaN grown on sapphire at UCSB using atmospheric-pressure MOCVD with a standard 2-step process [10]. We have found that the homoepitaxial growth mode observed depends not only on the temperature, growth rate, and other growth conditions, but also on the type of template used. Templates exhibiting two distinct behaviors have been found: ones on which all three classical homoepitaxial growth modes are observed (step-flow, layer-by-layer, and 3D) as temperature is lowered, and ones which show a direct transition from step-flow to 3-D, with no intervening layer-by-layer mode. For clarity we will refer to these as "type 1" and "type 2" templates, respectively. Although efforts are still underway to determine which property of the substrate (such as dislocation density) affects the transition [11], the direct step-flow to 3-D transition found for type 2 templates appears to be most representative of the behavior of high-quality GaN obtained from a variety of sources. 


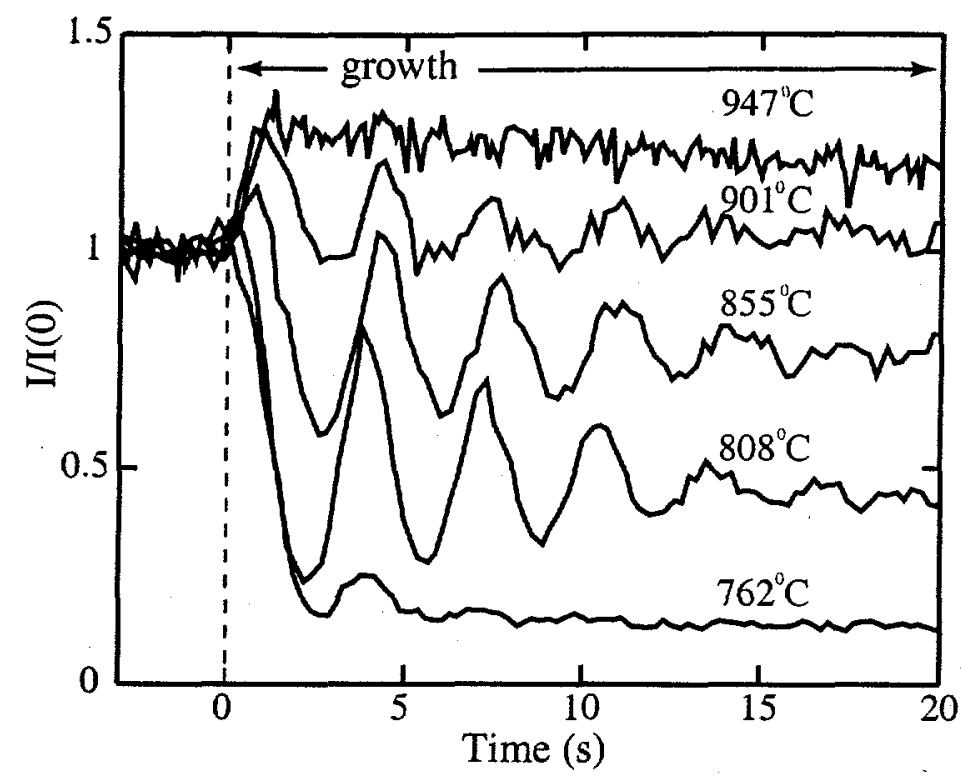

FIG. 2. Evolution of the $11 \overline{2} L$ CTR intensity at $L=0.95$ before and during $0.33 \mathrm{ML} / \mathrm{s}$ growth on a type 1 template at various temperatures. (After ref. [4].)

\section{LAYER-BY-LAYER GROWTH}

On some templates, we observe oscillations of the CTR intensity corresponding to layer-by-layer growth over a region of MOCVD growth conditions [4]. Observations of RHEED oscillations indicating layer-by-layer mode during MBE growth of GaN have likewise been reported by several groups [12]. Figure 2 shows the behavior of the CTR intensity during growth on such a type 1 template, at various temperatures for a constant TMG flow. Typical conditions for these growth runs are a total pressure of 200 Torr, a partial pressure of $\mathrm{NH}_{3}$ of 4.4 Torr, a TMG flow of $0.63 \mu \mathrm{mole} / \mathrm{min}$, and a total non-purge flow of $4.55 \mathrm{slpm}$, giving a V/III ratio of $7.1 \times 10^{3}$. For each temperature, a growth pulse is initiated by switching the TMG flow into the reactive gas stream. Growth starts when the TMG arrives at the sample, reaching steady-state within one second. From Fig. 2 one can see that the CTR oscillation amplitude varies as the growth mode changes from step-flow at high temperature, through layer-bylayer, to 3-D at low temperature. After growth is stopped by switching the TMG flow to vent, the surface gradually recovers its initial smoothness, and the CTR intensity increases back to its original value. The rate of recovery can be increased by heating the sample (e.g. to $950^{\circ} \mathrm{C}$ ). By performing successive growth and recovery cycles, it is possible to quickly investigate a variety of growth conditions using the same sample. The CTR oscillations observed with type 1 templates enable the mapping of the growth rate and growth mode boundaries as a function of process parameters [4]. We find that the growth rate is temperature-independent and TMG-transport-limited over a wide range of conditions. Both growth rates and the growth-mode transition are only weakly dependent on V/III ratio for values from $7.1 \times 10^{3}$ to $2.2 \times 10^{5}$. 


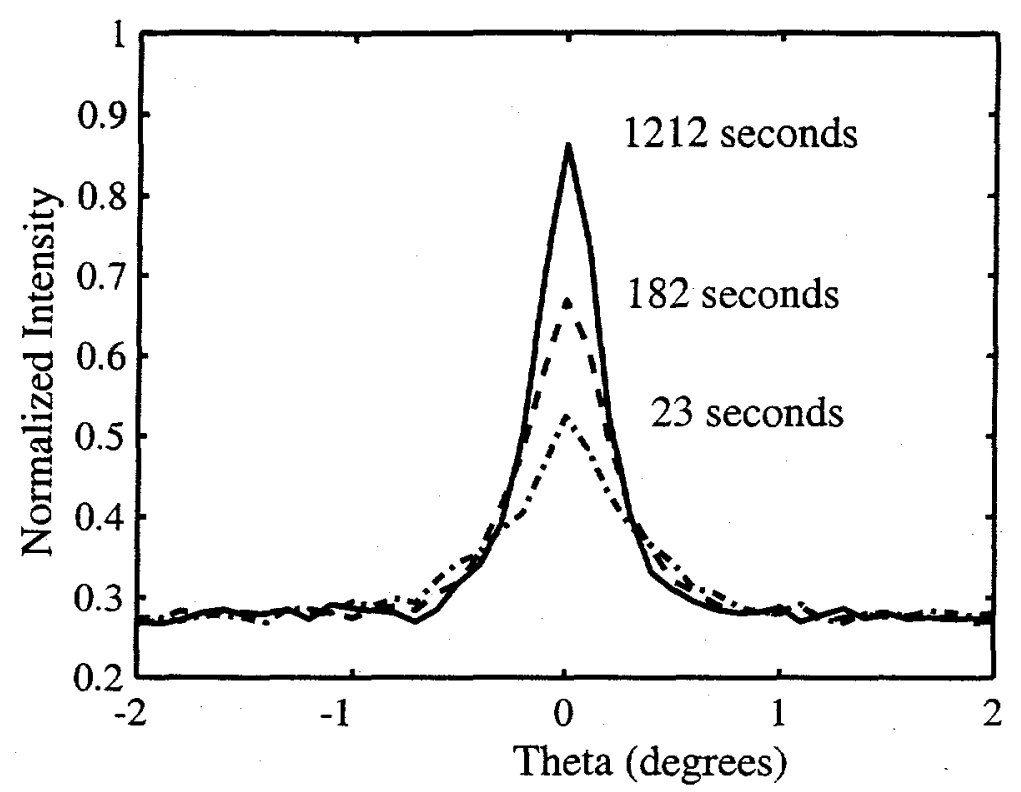

FIG. 3. Theta scans through the $11 \overline{2} L$ CTR at $L=0.9$ after growth of $1 / 2 \mathrm{ML}$ at $0.25 \mathrm{ML} / \mathrm{s}, 4.4$ Torr $\mathrm{NH}_{3}$, on a type 1 template, during island coarsening at 140 Torr $\mathrm{NH}_{3}$, $762^{\circ} \mathrm{C}$.

\section{E. ISLAND COARSENING}

We have also performed an experiment to investigate the rate of coarsening of islands on the GaN surface as a function of temperature and ammonia pressure. For this experiment, islands are formed by depositing 1/2 $\mathrm{ML}$ of $\mathrm{GaN}$ at a temperature in the layer-by-layer regime. These islands then grow through a coarsening process, eventually returning the surface to its initial smooth state. The rate of island coarsening following growth is monitored by observing the recovery of the CTR peak intensity, which is a function of the island size. This can be seen in Fig. 3, which shows transverse scans through the CTR at various times during island coarsening. When the islands are small, the CTR profile has a broad component; as the island size increases, the profile becomes sharper and has a higher peak intensity. The initial width of the broad component indicates an initial island size of $\sim 85 \AA$ for the growth conditions used.

Figure 4 shows the evolution of the CTR peak intensity during island coarsening under various conditions. In all cases, the islands are formed by deposition of $1 / 2 \mathrm{ML}$ at a fixed rate of $0.10 \mathrm{ML} / \mathrm{s}$ and fixed $\mathrm{p}\left(\mathrm{NH}_{3}\right)$ of 4.4 Torr. The larger initial drop in the CTR intensity as temperature is lowered is a consequence of the smaller initial island size produced at lower temperature. The generally higher coarsening rate at higher temperature, for a given island size, reflects the higher surface diffusivity. The effect of ammonia pressure on island coarsening has been determined by changing $\mathrm{p}\left(\mathrm{NH}_{3}\right)$ to 140 Torr within a few seconds after growth, for one run at each temperature. This assures that the initial island distribution is the same for both pressures at a given temperature, and that only the coarsening process is affected. Interestingly, the effect of increasing $\mathrm{p}\left(\mathrm{NH}_{3}\right)$ from 4.4 to 140 Torr depends on temperature; it 


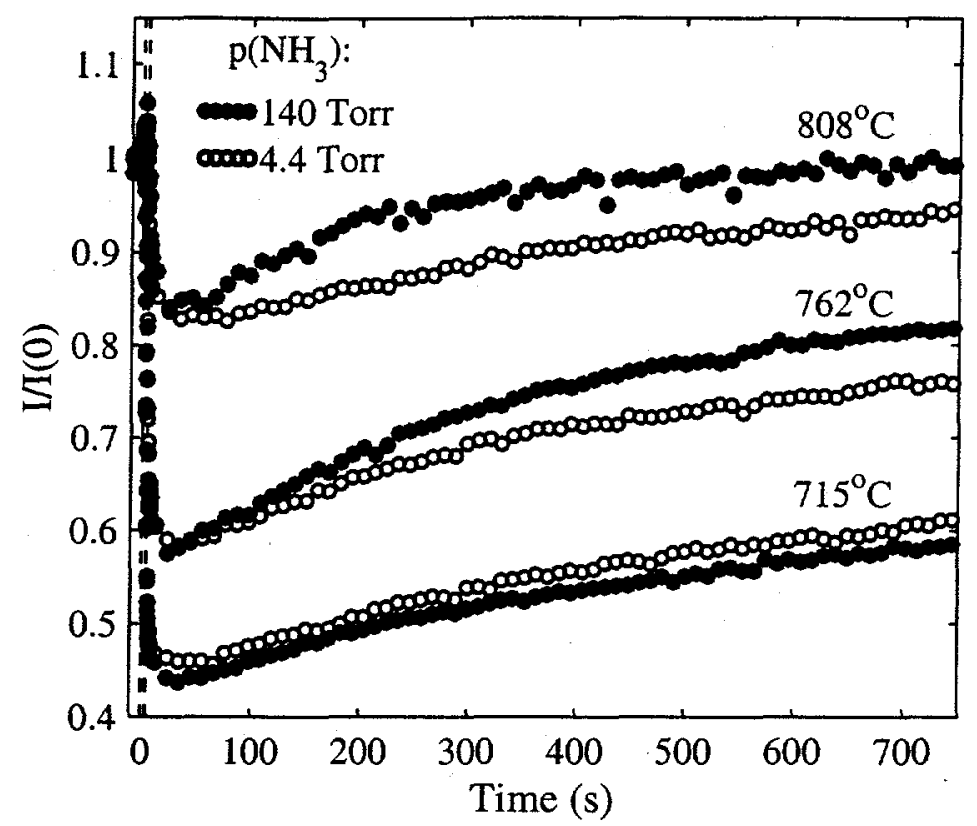

FIG. 4. Evolution of the $11 \overline{2} L$ CTR intensity at $L=0.9$ after growth of $1 / 2 \mathrm{ML}$ at 0.10 $\mathrm{ML} / \mathrm{s}, 4.4$ Torr $\mathrm{NH}_{3}$, on a type 1 template, during island coarsening at various temperatures and ammonia pressures.

changes the coarsening rate by factors of $0.95,1.6$, and 2.6 at $715^{\circ} \mathrm{C}, 762^{\circ} \mathrm{C}$, and $808^{\circ} \mathrm{C}$, respectively. Thus, at higher temperatures, a higher nitrogen potential in the MOCVD environment apparently increases mobility on the surface. This is in contrast to the effect inferred for the MBE environment, where a higher nitrogen potential is believed to give lower mobility [13].

\section{F. DIRECT STEP-FLOW TO 3-D TRANSITION}

In the majority of templates we have now studied, we observe a direct transition from step-flow to 3-D growth, with no intervening layer-by-layer mode. Figure 5 shows the evolution of the $11 \overline{2} L$ CTR intensity with time during undoped homoepitaxy of $\mathrm{GaN}$ on such a type 2 template, at various temperatures for the same fixed growth rate (6.5 ML in 20 seconds) used for the type 1 templates shown in Figure 2. Typical conditions for the growth runs discussed in this section are a total pressure of 200 Torr, a TMG flow of $0.78 \mu \mathrm{mole} / \mathrm{min}$, a partial pressure of $\mathrm{NH}_{3}$ of $38 \mathrm{Torr}$, and a total nonpurge flow of $6.25 \mathrm{slpm}$, giving a V/III ratio of $1.8 \times 10^{4}$. Above $800^{\circ} \mathrm{C}$, the behavior is independent of temperature, and indicates a step-flow growth mode. The increase in the CTR intensity during step-flow growth indicates a change in the surface structure relative to the equilibrium (pre-growth) structure of $\mathrm{GaN}(0001)$ in $\mathrm{NH}_{3}$ [3], which we attribute to a reduction in the density of Ga vacancies in the surface layer. The CTR intensity decays monotonically during growth, and the decay rate increases significantly as temperature is decreased below $800^{\circ} \mathrm{C}$. This roughening is the onset of a transition in the growth mode from step-flow directly to $3-\mathrm{D}$ growth. Note that the growth mode is predominantly step-flow over the whole temperature range 


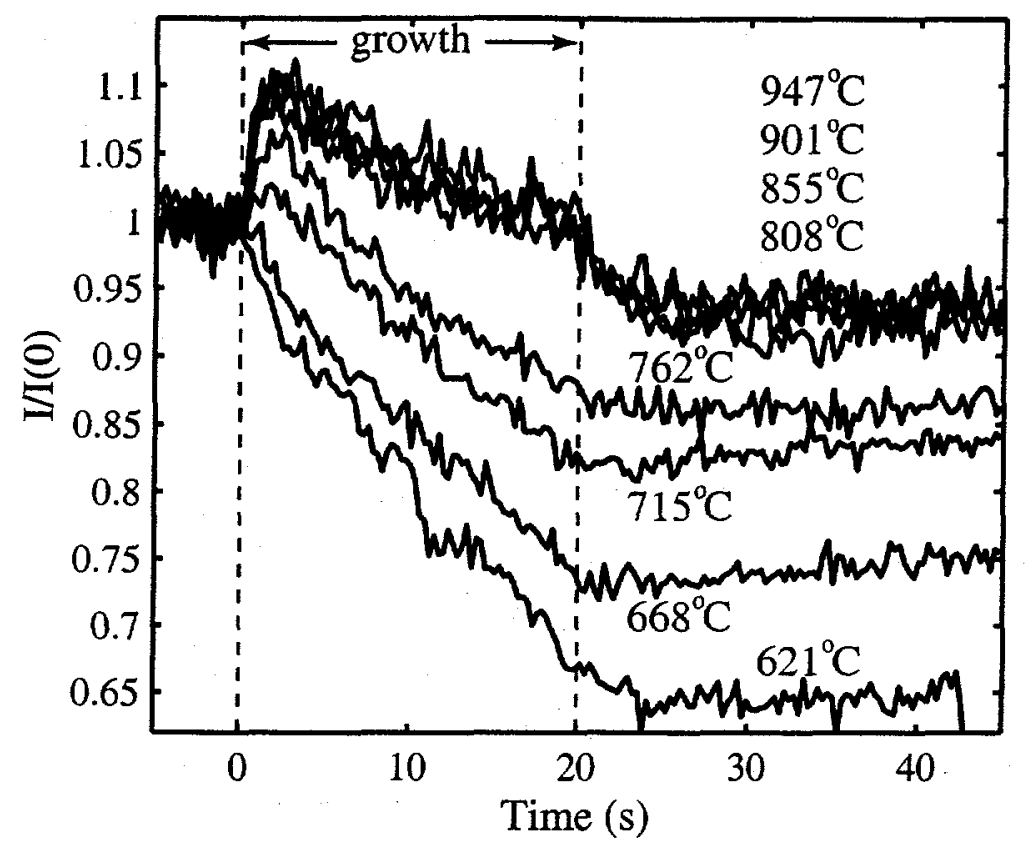

FIG. 5. Evolution of the $11 \overline{2} L$ CTR intensity at $L=0.9$ before, during, and after 0.33 $\mathrm{ML} / \mathrm{s}$ growth on a type 2 template at various temperatures.

investigated; even at the lowest temperature $\left(620^{\circ} \mathrm{C}\right)$, the roughening rate indicated by the CTR intensity decay is only $1.5 \%$ of that for Poisson-limited 3-D growth [14,15].

\section{G. EFFECTS OF IMPURITIES}

In additional experiments we have studied the effects of silicon and oxygen impurities on the growth modes of GaN films deposited on type 2 templates. Silicon is a commonly used $n$-type dopant in $\mathrm{GaN}$, which has been shown to produce a smoother surface morphology for low temperature $\left(\sim 800^{\circ} \mathrm{C}\right)$ growth [16]. However, it has been reported that $\mathrm{Si}$ concentrations above $1 \times 10^{19} \mathrm{~cm}^{-3}$ induce surface roughening [17]. Our study of oxygen, a common unintentional impurity, has been undertaken in an effort to understand whether potential contamination levels could be affecting our results.

We have investigated the effect of Si impurity concentration on growth of GaN by observing the evolution of the CTR during growth pulses at various temperatures, with increasing Si concentrations. However, we find that the growth behavior depends at least as much on the length of time since prior growth with $\mathrm{Si}$ as it does on the current $\mathrm{Si}$ concentration. In particular, we find that if a layer with a high concentration of $\mathrm{Si}$ is annealed, the layer-by-layer growth mode is induced in subsequent growth [6]. In order to understand on this phenomenon, we have introduced Si directly onto the surface by exposing the sample to a dose of disilane, followed by a growth pulse without Si identical to those shown in Fig. 5. The results are shown in Fig. 6 for various $\mathrm{Si}$ doses at $901^{\circ} \mathrm{C}$. The Si doses are calculated assuming the 


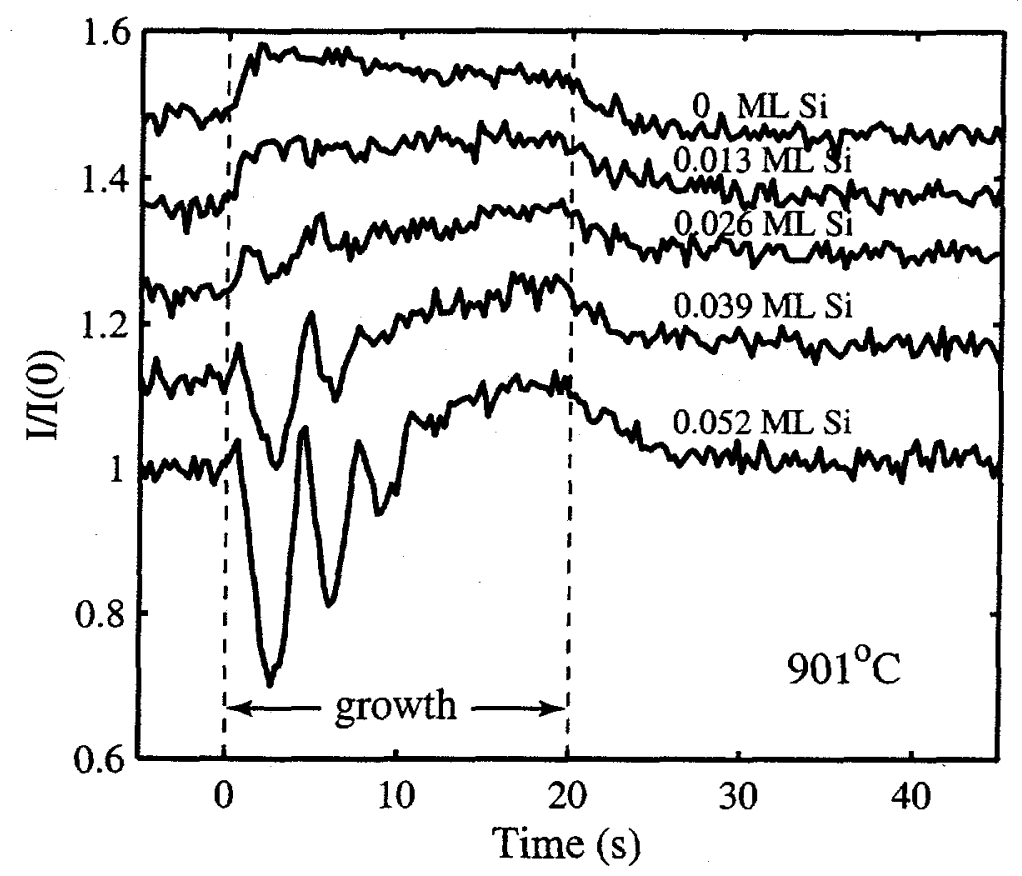

FIG. 6. Evolution of the $11 \overline{2} L$ CTR intensity at $L=0.9$ before, during, and after undoped $0.33 \mathrm{ML} / \mathrm{s}$ growth on a type 2 template at $901^{\circ} \mathrm{Cafter}$ various surface doses of Si.

same incorporation efficiency for a given disilane flow as found for growth of a film containing $\mathrm{Si}$, for which the $\mathrm{Si}$ concentration in the film has a linear dependence on the gas-phase [Si]/[Ga] ratio [18]. We find that a recovery cycle of growth without $\mathrm{Si}$ can be used to bury the Si and return the sample surface to an initial state without impurities having consistent morphology, so that the same sample can be used to study a series of conditions. From the data in Fig. 6, one can see that a surface dose of $0.026 \mathrm{ML}$ of $\mathrm{Si}$ produces weak oscillations in the CTR intensity, and as the dose is increased up to $0.052 \mathrm{ML}$ the layer-by-layer growth mode becomes more dominant. Thus, sufficient silicon on the surface induces layer-by-layer growth under conditions which would otherwise produce the step-flow mode. Since this same effect is obtained when a layer containing $\mathrm{Si}$ is annealed, $\mathrm{Si}$ presumably segregates to the surface to induce layer-by-layer growth [6].

In contrast to silicon, the effect of oxygen on the growth mode is relatively weak. Figure 7 shows the evolution of the CTR intensity during growth at $715^{\circ} \mathrm{C}$ for various concentrations of $\mathrm{O}_{2}$ added to the total non-purge flow. Concentrations up to a few parts per million have no measurable effect on the growth mode, while significantly higher concentrations increase the roughening rate during low-temperature growth. No tendency to induce layer-by-layer growth is observed, and the same procedure (heating to $950^{\circ} \mathrm{C}$ ) used without impurities to recover the surface to its smooth, pregrowth state is also found to work in the presence of $\mathrm{O}_{2}$. Note that the concentration of $\mathrm{O}_{2}$ needed to affect the growth mode is quite large; $190 \mathrm{ppm}$ corresponds to a gasphase $\mathrm{O} / \mathrm{Ga}$ ratio during growth of 136 . These concentrations are much higher than the background levels expected to be present given the purified sources and purging procedures we use $(<<1 \mathrm{ppm})$, indicating that unintentional $\mathrm{O}_{2}$ contamination does not affect our growth mode results. 


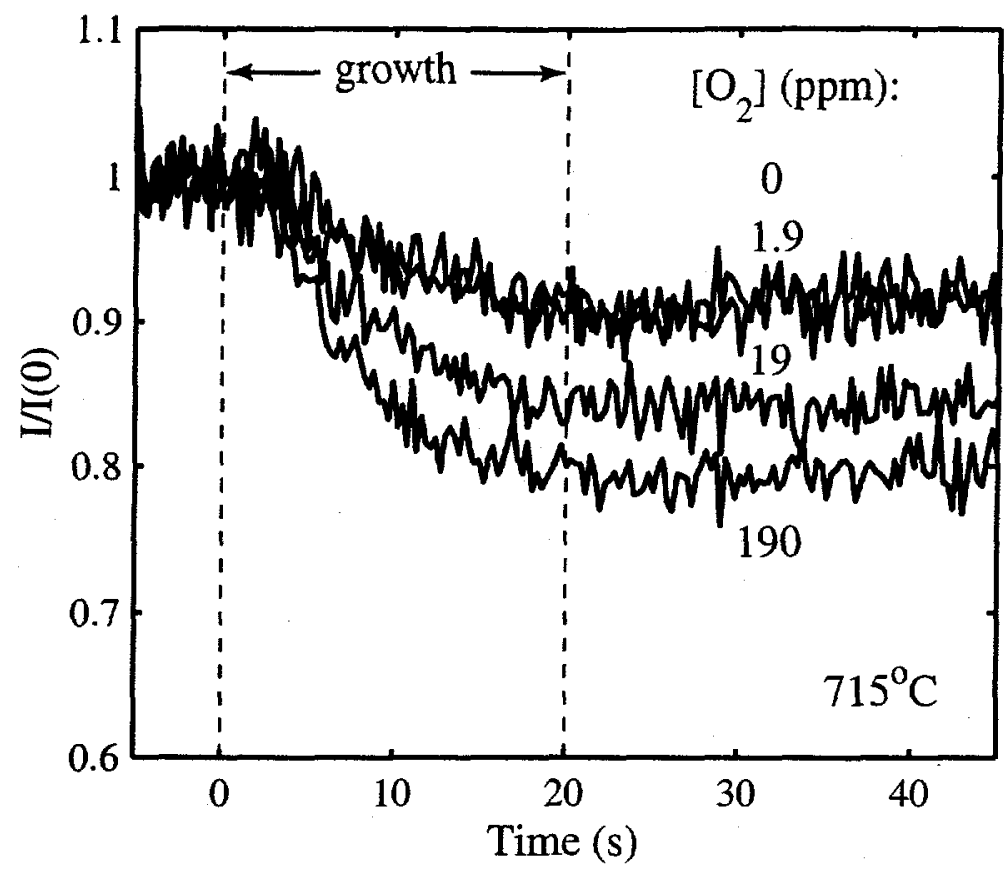

FIG. 7. Evolution of the $11 \overline{2} L$ CTR intensity at $L=0.9$ before, during, and after 0.33 $\mathrm{ML} / \mathrm{s}$ growth on a type 2 template at $715^{\circ} \mathrm{C}$ for various oxygen doping levels.

\section{H. DISCUSSION AND CONCLUSIONS}

We have found two types of growth mode behavior for MOCVD growth of GaN, characterized by whether or not deposition conditions exist under which CTR oscillations corresponding to layer-by-layer growth occur. We have verified that this difference is determined by the template used for growth, although many properties of the two types of templates, such as crystal mosaic spread and surface step density, appear to be similar. Work is in progress to determine which properties of the substrate (such as dislocation type and density) may determine the growth mode transition behavior [11]. Our experience indicates that the direct step-flow to 3-D transition is most representative of the behavior of high-quality GaN obtained from a variety of sources. Templates which do exhibit layer-by-layer growth provide a particularly good opportunity for detailed GIXS studies of the effects of environmental conditions on surface dynamics such as island coarsening.

The direct step-flow to 3-D growth mode transition observed for undoped growth on type 2 templates of $\mathrm{GaN}(0001)$ is similar to the growth mode transitions seen on close-packed metal surfaces such as $\operatorname{Ag}(111)$ [15]. On many pure metals, the closepacked surface exhibits a direct step-flow to $3-\mathrm{D}$ growth-mode transition. This has been explained in terms of a low ratio of the activation barrier for surface diffusion to the activation barrier for transport over a step edge, which restricts interlayer transport and suppresses the island coalescence needed for layer-by-layer growth, leading to mound formation [15]. These considerations may also apply to $\mathrm{GaN}(0001)$, which is likewise a close-packed surface. In particular, the direct step-flow to 3-D transition may be due to a relatively low surface diffusion activation barrier. However, 
an alternative roughening mechanism that must be considered for GaN films, which typically have threading dislocation densities greater than $10^{8} \mathrm{~cm}^{-2}$, is the pinning of steps at threading dislocations. Such surface morphologies have been observed for low-temperature MOVPE growth [16].

The ability of $\mathrm{Si}$ to induce layer-by-layer growth on $\mathrm{GaN}(0001)$ is likewise similar to effects of surfactants on close-packed metal surfaces such as $\mathrm{Sb}$ on $\mathrm{Ag}(111)$ [19]. It has been proposed that surfactants induce layer-by-layer growth on close-packed metal surfaces by changing the relative rates of various competing processes: surface diffusion on the terraces, diffusion across steps, attachment of adatoms at steps, and island nucleation [20]. However, while surfactants induce layer-by-layer growth in the 3-D regime of metal (111) surfaces, we find that $\mathrm{Si}$ induces layer-by-layer growth in the step-flow regime of $\mathrm{GaN}(0001)$ at temperatures as high as $990^{\circ} \mathrm{C}$ ) [6]. The best explanation for this behavior may be theories of "impurity poisoning" of growth due to the pinning of step motion by impurity barriers [21]. These predict an increase in the adatom supersaturation at a given growth rate, which would enhance the island nucleation rate and favor layer-by-layer growth.

The results presented here provide examples of ways in which in situ $\mathrm{x}$-ray scattering studies of surface morphology during MOCVD growth can give insight into the mechanisms of growth and the interactions between the vapor-phase environment and the crystal surface. By expanding the facilities for such studies at the BESSRC-CAT beamlines at the Advanced Photon Source, we plan to continue studies of GaN-based materials as well as begin studies of CVD growth of complex oxides.

\section{ACKNOWLEDGEMENTS}

This work is supported by the National Science Foundation under grant DMR9704201, the State of Illinois under HECA, and the U.S. Department of Energy, BES-DMS under contract W-31-109-ENG-38. 


\section{REFERENCES}

[1] J.Y. Tsao, Materials Fundamentals of Molecular Beam Epitaxy, pp. 236-258, Academic Press, San Diego, 1993.

[2] A. Munkholm, G.B. Stephenson, J.A. Eastman, C. Thompson, P. Fini, J.S. Speck, O. Auciello, P.H. Fuoss, and S.P. DenBaars, Phys. Rev. Lett., 83, 741 (1999).

[3] A. Munkholm, C. Thompson, G.B. Stephenson, J.A. Eastman, O. Auciello, P. Fini, J.S. Speck, and S.P. DenBaars, Physica B, 283, 217 (2000).

[4] G.B. Stephenson, J.A. Eastman, C. Thompson, O. Auciello, L.J. Thompson, A. Munkholm, P. Fini, S.P. DenBaars, and J.S. Speck, Appl. Phys. Lett., 74, 3326 (1999).

[5] M.V. Ramana Murty et al., to be published.

[6] A. Munkholm et al., to be published.

[7] P. Fini, A. Munkholm, Carol Thompson, G.B. Stephenson, J.A. Eastman, M.V. Ramana Murty, O. Auciello, L. Zhao, S.P. DenBaars, and J.S. Speck, to appear in Appl. Phys. Lett., (2000).

[8] G.B. Stephenson, J.A. Eastman, O. Auciello, A. Munkholm, C. Thompson, P.H. Fuoss, P. Fini, S.P. DenBaars, and J.S. Speck, MRS Bull., 24 (1), 21 (January 1999).

[9] P. H. Fuoss and S. Brennan, Annu. Rev. Mater. Sci., 20, 365 (1990); I. K. Robinson and D. J. Tweet, Rep. Prog. Phys., 55, 599 (1992).

[10] X.H. Wu, P. Fini, S. Keller, E.J. Tarsa, B. Heying, U.K. Mishra, S.P. DenBaars, and J.S. Speck, Jpn. J. Appl. Phys., 35, L1648 (1996).

[11] J.A. Eastman et al., to be published.

[12] N. Grandjean, J. Massies, P. Vennegues, M. Leroux, F. Demangeot, M. Renucci, and J. Frandon, J. Appl. Phys., 83, 1379 (1998); B. Daudin and F. Widmann, J. Cryst. Growth, 182, 1 (1997).

[13] E.J. Tarsa, B. Heying, X.H. Wu, P. Fini, S.P. DenBaars, and J.S. Speck, J. Appl. Phys., 82, 5472 (1997).

[14] A. Zangwill, J. Cryst. Growth, 163, 8 (1996).

[15] W.C. Elliott, P.F. Miceli, T. Tse, and P.W. Stephens, Phys. Rev. B, 54, 17938 (1996).

[16] S. Keller, S.F. Chichibu, M.S. Minsky, E. Hu, U.K. Mishra, and S.P. DenBaars, J. Cryst. Growth, 195, 258 (1998).

[17] T.S. Cheng, in Properties, Processing, and Applications of GaN and Related Semiconductors, J.H Edgar, S. Strite, I. Akasaki, and H. Amano, eds., p. 349, INSPEC, London (1999).

[18] G. Parish, S. Keller, S.P. DenBaars, and U.K. Mishra, J. Elec. Mat., 29, 15 (2000).

[19] H.A. van der Vegt, H.M. van Pinxteren, M. Lohmeier, E. Vlieg, and J.M.C. Thornton, Phys. Rev. Lett., 68, 3335 (1992).

[20] H.A. van der Vegt, J. Vrijmoeth, R.J. Behm, and E. Vlieg, Phys. Rev. B, 57, 4127 (1998).

[21] N. Cabrera and D.A. Vermilyea, in Growth and Perfection of Crystals, R.H. Doremus, B.W. Roberts, and D. Turnbull, eds., p. 393, Chapman and Hall, London (1958); K. Sangwal and T. Pałczyńska, J. Cryst. Growth, 212, 522 (2000). 